\title{
REMARKS ON DERIVATIONS ON SEMIPRIME RINGS
}

\author{
MOHAMAD NAGY DAIF \\ Department of Mathematics \\ Faculty of Education \\ Umm A1-Qura University \\ Taif, Saudi Arabia \\ and \\ HOWARD E. BELL \\ Department of Mathematics \\ Brock University \\ St. Catharines, Ontario \\ Canada L2S 3A1 \\ (Received December 31, 1990 and in revised form May 10, 1991)
}

ABSTRACT. We prove that a semiprime ring $R$ must be commutative if it admits a derivation $d$ such that $(i) x y+d(x y)=y x+d(y x)$ for all $x, y$ in $R$, or (ii) $x y-d(x y)=$ $y x-d(y x)$ for all $x, y$ in $R$. In the event that $R$ is prime, (i) or (ii) need only be assumed for all $x, y$ in some nonzero ideal of $R$.

KEY WORDS AND PHRASES. Derivation, semiprime ring, prime ring, commutative, central ideal, integral domain, direct sum.

1980 AMS SUBJECT CLASSIFICATION CODES. 16A15, 16A70.

1. INTRODUCTION.

In the past fifteen years, there has been an ongoing interest in derivations on prime or semiprime rings; and many of the results have involyed commutativity. (See [1] for a partial bibliography.) In this brief note, we explore the commutativity implications of the existence on $R$ of a derivation $d$ satisfying the following:

(*) there exists a nonzero ideal $K$ of $R$ such that either $x y+d(x y)=y x+d(y x)$ for all $x, y$ in $K$, or $x y-d(x y)=y x-d(y x)$ for all $x, y$ in $K$.

2. THE PRINCIPAL RESULTS.

Our principal results in this note are

THEOREM 1. If $R$ is any prime ring admitting a derivation d satisfying (*), then $R$ is commutative.

THEOREM 2. Let $R$ be a semiprime ring admitting a derivation $d$ for which either $x y+d(x y)=y x+d(y x)$ for all $x, y$ in $R$ or $x y-d(x y)=y x-d(y x)$ for all $x, y$ in $R$. Then $R$ is commutative.

In fact, both of these theorems are consequences of a third theorem, which is reminiscent of the results in [1]. 
THEORLM 3. If $R$ is a semiprime ring admitting a derivation d satisfying (*), the $K$ is a central ideal.

3. PROOFS.

The proof of Theorem 3 hinges on the following lemma.

LEMMIA 1. Let $R$ be a semiprime ring and $I$ a nonzero ideal of $R$. If $z$ in $R$ centralizes the set $[I, I]$, then $z$ centralizes $I$.

l'ROOF. Let $z$ centralizes $[I, I]$. Then for all $x, y$ in $I$, we have $z[x, x y]=[x, x y] z$, which can be rewritten as $z x[x, y]=x[x, y] z$; hence $[z, x][x, y]=0$ for all $x, y$ in $I$. Replacing y by $y z$, we get $[z, x] I[z, x]=\{0\}$. Since $I$ is an ideal, it follows that, $[z, x] \operatorname{IR}[z, x] I=\{0\}=\operatorname{I}[z, x] R I[z, x]$, so that $[z, x] I=I[z, x]=\{0\}$. Thus, $[[z, x], x]=0$ for all $x$ in I; and by Theorem 3 of [2], z centralizes $I$.

For ease of reference, we include a second lemma, which is wel1-known.

LEMIM 2. (a) If $R$ is a prime ring with a nonzero central ideal, then $R$ is commutative.

(b) If $R$ is a semiprime ring, the center of a nonzero ideal is contained in the center of $R$.

PROOF OF THEOREM 3. We suppose first that

$$
x y+d(x y)=y x+d(y x) \text { for } a 11 x, y \text { in } K,
$$

which can be rewritten as

$$
[x, y]=-d([x, y]) \text { for all } x, y \text { in } K \text {. }
$$

Now for all $x, y, z$ in $K$, we have $[x, y] z+d([x, y] z)=z[x, y]+d(z[x, y])$, which yields $[x, y] z+d([x, y]) z+[x, y] d(z)=z[x, y]+d(z)[x, y]+z d([x, y]) ;$

and applying (2) we conclude thạt

$$
[x, y] d(z)=d(z)[x, y] \text { for a11 } x, y, z \text { in } K \text {. }
$$

By Lemma 1, we see that $d(K)$ centralizes $K$; and it follows from (1) that $[x, y]$ is in the center of $\mathrm{K}$ for $\mathrm{all} \mathrm{x}, \mathrm{y}$ in $\mathrm{K}$. Another application of Lemma 1 shows that the ideal $K$ is commutative; hence by Lemma $2(b), K$ is in the center of $R$. In the event that $x y-$ $d(x y)=y x-d(y x)$ for all $x, y$ in $K$, it is equally easy to establish ( 3$)$, therefore our proof is complete.

Theorem 2 is immediate from Theorem 3, and Theorem 1 follows from Theorem 3 and Lemma $2(a)$.

We remark, in conclusion, that under the hypotheses of Theorem 3 we cannot hope to prove commutativity of $R$. Consider $R=R_{1} \oplus R_{2}$, where $R_{1}$ is an integral domain, $R_{2}$ is a prime ring which is not commutative, and $d$ is the "direct sum" of derivations on the summands $R_{1}$ and $R_{2}$.

ACKNOWLEDGEMENT. H. H. Bell was supported by the Natural Sciences and Engineering Research Council of Canada, Grant No. A3961.

\section{REFERENCES}

1. BELL, H.E. and MARTINDALE III, W.S. Centralizing Mappings of Semiprime Rings, Canad. Math. Bul1. 30 (1987), 92-101.

2. BELL, H.E. and NADA, I. On Some Center-like Subsets of Rings, Arch. Math. 48 $(1987), 381-387$. 


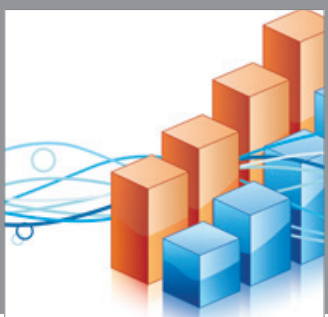

Advances in

Operations Research

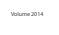

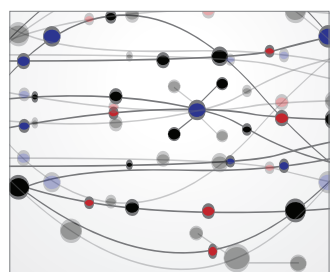

\section{The Scientific} World Journal
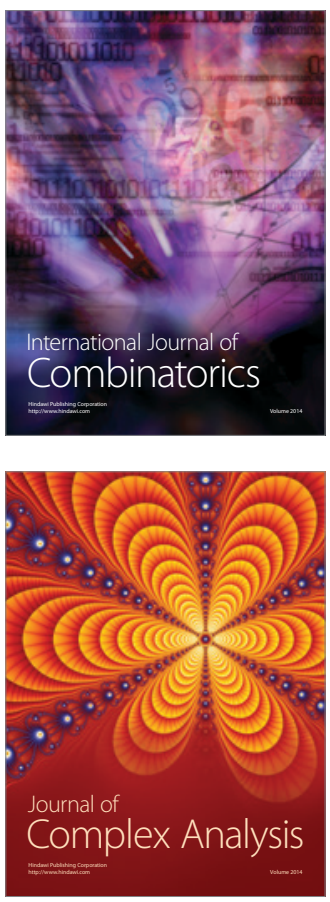

International Journal of

Mathematics and

Mathematical

Sciences
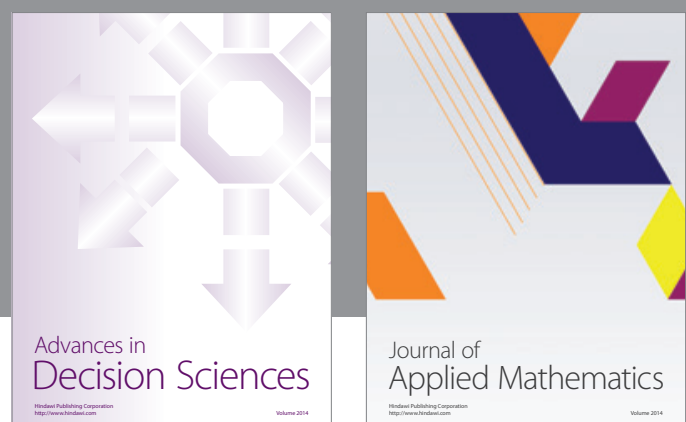

Journal of

Applied Mathematics
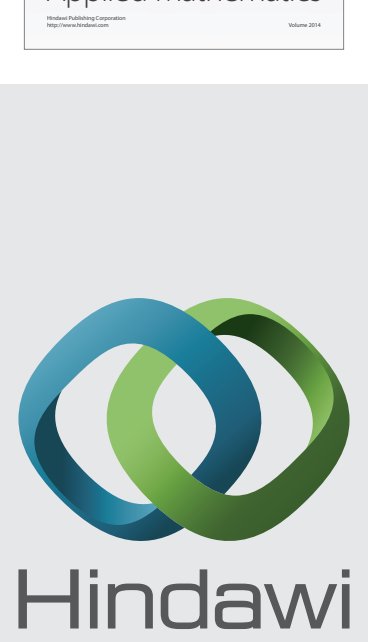

Submit your manuscripts at http://www.hindawi.com
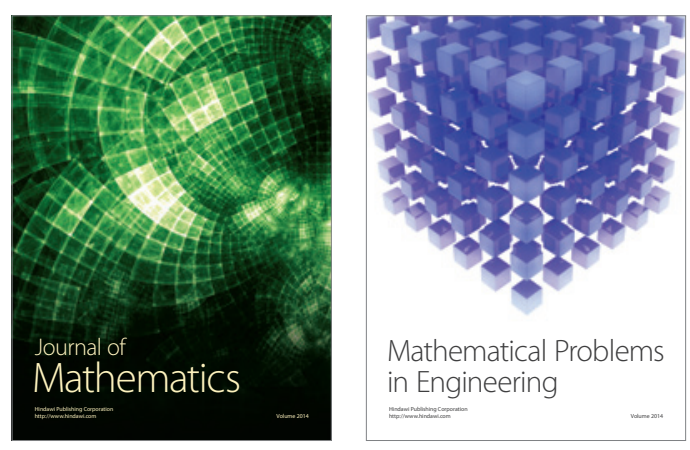

Mathematical Problems in Engineering
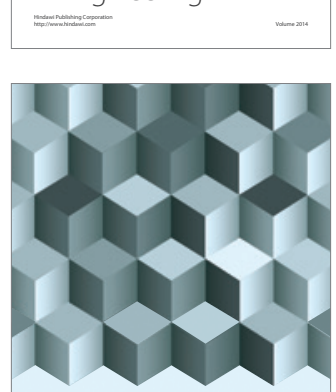

Journal of

Function Spaces
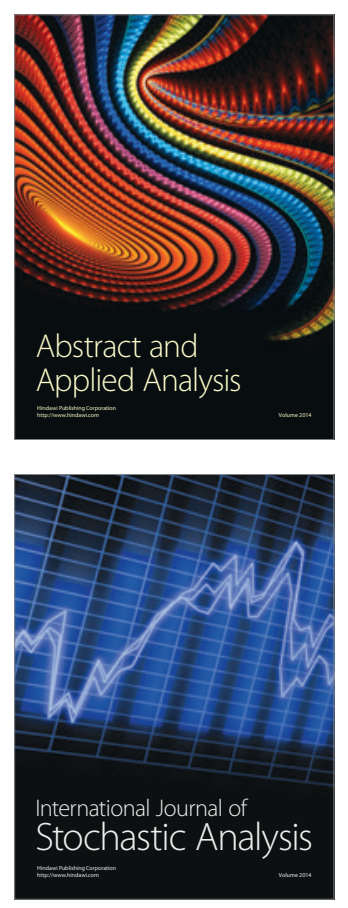

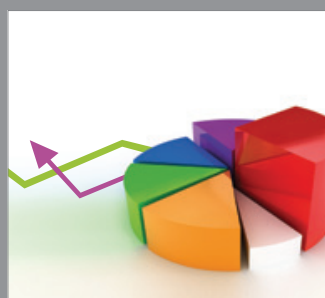

ournal of

Probability and Statistics

Promensencen
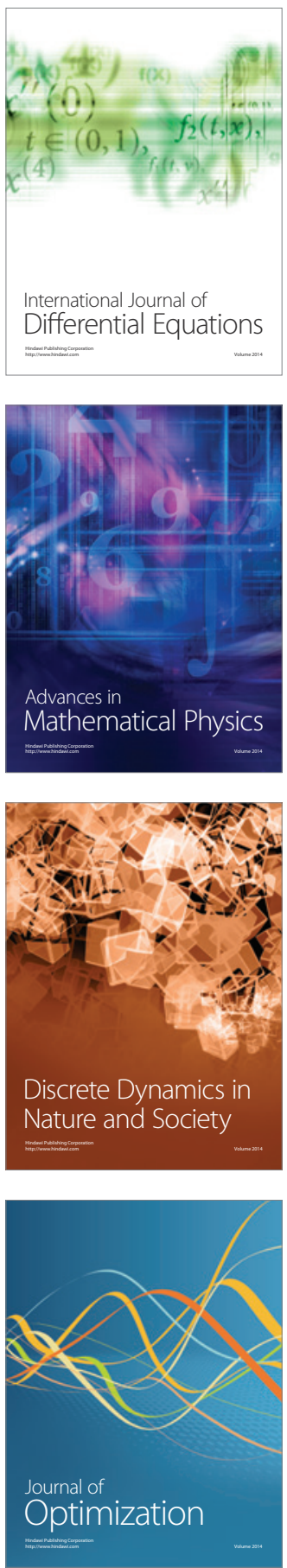